\title{
A THEOREM ON INFINITE NIELSEN EXTENSIONS
}

\author{
NOEMI HALPERN
}

\begin{abstract}
It is shown that every Riemann surface with $n$ punctures is the infinite Nielsen extension of a surface with $n$ holes.
\end{abstract}

Let $S_{0}$ be a Riemann surface of genus $g$ with $n$ punctures and $m$ holes. Assume that $6 g-6+2 n+3 m>0$ and that $m>0$. Then $S_{0}$ can be represented as $U / G$ where $U$ is the upper half-plane and $G$ is a torsion-free Fuchsian group of the second kind. There is an infinite set $I$ of open intervals of $R \cup\{\infty\}$ on which $G$ acts discontinuously. Let $\Omega=U \cup L \cup I$, where $L$ denotes the lower half-plane. The quotient $S_{0}^{d}=\Omega / G$ is the double of $S_{0}$. It is a compact surface, except for finitely many punctures. The surface $S_{0}^{d}$ can be represented as $U / G^{\prime}$, where $G^{\prime}$ is a torsion-free Fuchsian group of the first kind. Let $D$ be a component of the preimage of $S_{0}$ under the natural projection $U \rightarrow U / G^{\prime}$ and let $G_{1}$ be the stabilizer of $D$ in $G^{\prime}$. Then the surface $S_{1}=U / G_{1}$ is the Nielsen extension of $S_{0}$. Given a surface $S_{0}$, let $S_{k}$ be the Nielsen extension of $S_{k-1}$, for $k \in N$. Define the infinite Nielsen extension of $S_{0}$ to be $S_{\infty}=S_{0} \cup S_{1} \cup S_{2} \cup \cdots$.

Bers has conjectured that given a Riemann surface $S$ of type $(g ; n, 0)$ and positive numbers $l_{1}, \ldots, l_{n}$ there exists a surface of type $(g ; 0, n)$ with boundary elements of lengths $l_{1}, \ldots, l_{n}$ such that its infinite Nielsen extension is $S$ (oral communication). This conjecture was proven in [4] for a torus with a hole, i.e. when $g=n=1$. In this paper it will be proven for all $g$ but without being able to specify the lengths of the holes. The Poincaré metric is used and the length of a boundary curve refers to the Poincare length of the geodesic to which it is freely homotopic.

THEOREM. Given a surface $S$ of type $(g ; n, 0)$ there exists a surface of type $(g ; 0, n)$ such that its infinite Nielsen extension is $S$.

The following inequalities concerning the lengths of geodesics of Nielsen extensions are need in the proof. They are proven in [3].

I. Given a finite Riemann surface with a boundary curve of length $l$, then the length of the corresponding boundary curve of its Nielsen extension is less than $\frac{1}{2} l$. The length of the corresponding boundary element of its infinite Nielsen extension is therefore zero.

II. Let $S$ be a finite Riemann surface such that all its boundary curves have length less than $l$. Assume that $S$ has a simple closed geodesic of length $x$ not homotopic to a boundary curve. Denote by $x_{n}\left(x_{\infty}\right)$ the length of the corresponding geodesic of its $n$th (its infinite) Nielsen extension. Then

$$
c x<x_{\infty}<x \text { and } c_{n} x_{n}<x_{\infty}<x_{n}
$$

Received by the editors January 18, 1985 and, in revised form, July 8, 1985.

1980 Mathematics Subject Classification. Primary 30F25, 30F35, 32G15. 
where

$$
c=\prod_{i=1}^{\infty}\left\{1-2 / \Pi \tan ^{-1} 2 \sinh l / 2^{i}\right\}
$$

and

$$
c_{n}=\prod_{i=n}^{\infty}\left\{1-2 / \Pi \tan ^{-1} 2 \sinh l / 2^{i}\right\} .
$$

Note that $c$ and $c_{n}$ are convergent infinite products which are close to 1 for small $l$ and $c_{n} \rightarrow 1$ as $n \rightarrow \infty$.

The following lemma will be used in the proof of the theorem:

LEMMA. For $k \geq 0$ and $n \geq 1$ let $\psi: R^{k} \times[0, \infty)^{n} \rightarrow R^{k} \times[0]^{n}$ be a continuous function such that its restriction to $R^{k} \times[0]^{n}$ is the identity map. Then the restriction of $\psi$ to $R^{k} \times(0, \infty)^{n}$ is a surjection onto $R^{k} \times[0]^{n}$.

To simplify notation, assume that the image space of $\psi$ is $R^{k}$; that is, $\psi: R^{k} \times$ $[0, \infty)^{n} \rightarrow R^{k}$.

ProOF. Assume that the lemma is false, i.e. that there exists a point $P \in$ $R^{k} \times[0]^{n}$ such that

$$
\psi\left(R^{k} \times(0, \infty)^{n}\right) \subset Y=R^{k}-\{P\} .
$$

Let $x \in R^{k}$ and $y \in[0, \infty)^{n}$ and define $\psi_{y}: R^{k} \rightarrow R^{k}$ by $\psi_{y}(x)=\psi(x, y)$. Let $y_{0}=[0]^{n}$. Then, by the hypothesis of the lemma, $\psi_{y_{0}}(x)=x$ for all $x$. Pick $R>\|P\|$ and let

$$
S=\left\{x \in R^{k} \mid\|x\|=R\right\} .
$$

Then $\psi_{y_{0}}: S \rightarrow Y$ represents a nontrivial element of $H_{k-1}(Y ; Z)$. For any point $y_{1} \in(0, \infty)^{n}$, the map $\psi_{y_{1}}: S \rightarrow Y$ is homotopic to $\psi_{y_{0}}$ and thus represents the same nontrivial element of $H_{k-1}(Y ; Z)$. Since $H_{k-1}\left(R^{k} ; Z\right) \simeq\{0\}, \psi_{y_{1}}: R^{k} \rightarrow Y$ induces the zero map

$$
\psi_{y_{1}}^{*}: H_{k-1}\left(R^{k} ; Z\right) \rightarrow H_{k-1}(Y ; Z) .
$$

But $\psi_{y_{1}}$ takes $S$ to a nontrivial element of $H_{k-1}(Y ; Z)$. This gives us a contradiction.

Given a topological orientable surface $S$ of genus $g$ with $n$ boundary elements, the Fricke space $F$ of $S$ is the set of homotopy equivalence classes of pairs $(h, R)$ where $h$ is an orientation preserving homeomorphism of $S$ onto a Riemann surface $R$.

THEOREM A [2]. The Fricke space $F$ is homeomorphic to $R^{k} \times[0, \infty)^{n}$, where $k=6 g-6+2 n$ and the last $n$ coordinates represent the lengths of the holes.

The topology on $F$ is the weakest topology which makes lengths of closed geodesics continuous. Denote by $x_{C}$ the length of a geodesic $C$.

THEOREM B [2]. Let $f: F \rightarrow F$ be a function such that $x_{C} \circ f$ is continuous for every simple closed geodesic $C$. Then $f$ is continuous.

PROOF OF THE THEOREM. Let $F$ denote the Fricke space of surfaces of genus $g$ with $n$ boundary elements. An element of $F$ will refer to a point of $R^{k} \times[0, \infty)^{n}$ or to the marked surface represented by the point. Denote by $F_{0}$ the subset $R^{k} \times[0]^{n}$ 
of $F$ and by $F_{1}$ the set $F-F_{0}$. The points of $F_{0}$ correspond to the surfaces with $n$ punctures; the points of $F_{1}$ correspond to those surfaces with at least one boundary curve of positive length. The topology is the usual one on surfaces with holes.

Let $f_{n}: F_{1} \rightarrow F_{1}$ be the map which takes a surface into its $n$th Nielsen extension. It will be shown to be continuous. Let $S \in F_{1}$ and $\left\{S_{m}\right\} \subset F_{1}$ such that $S_{m} \rightarrow S$. Then the doubles of $S_{m}$ converge to the double of $S$, i.e. $S_{m}^{d} \rightarrow S^{d}$.

There exist Fuchsian groups $G_{m}=\left\langle A_{1}^{m}, \ldots, A_{2 \alpha}^{m}\right\rangle$ and $G=\left\langle A_{1}, \ldots, A_{2 \alpha}\right\rangle$ with $\alpha=2 g+n-1$ such that

(i) $S_{m}^{d}=U / G_{m}$ and $S^{d}=U / G$,

(ii) $A_{i}^{m} \rightarrow A_{i}$ for $i=1, \ldots, 2 \alpha$,

(iii) the Nielsen extension of $S_{m}$ is $U /\left\langle A_{1}^{m}, \ldots, A_{\alpha}^{m}\right\rangle$, and

(iv) the Nielsen extension of $S$ is $U /\left\langle A_{1}, \ldots, A_{\alpha}\right\rangle$.

But then $U /\left\langle A_{1}^{m}, \ldots, A_{\alpha}^{m}\right\rangle$ converges to $U /\left\langle A_{1}, \ldots, A_{\alpha}\right\rangle$ and $f_{1}\left(S_{m}\right) \rightarrow f_{1}(S)$. Therefore, $f_{1}$ is continuous. Since the composition of continuous functions is continuous, the function $f_{n}=f_{1} \circ \cdots \circ f_{1}$ is also continuous.

Define the function $f_{\infty}: F \rightarrow F_{0}$ as follows:

$$
f_{\infty}(S)= \begin{cases}\lim _{n \rightarrow \infty} f_{n}(S) & \text { if } S \in F_{1}, \\ S & \text { if } S \in F_{0} .\end{cases}
$$

Clearly, $f_{n}(S)=S_{n} \subset S_{n+1}=f_{n+1}(S)$ and $\lim _{n \rightarrow \infty} f_{n}(S)=S_{0} \cup S_{1} \cup S_{2} \cup \cdots=$ $S_{\infty}$. Thus $f_{\infty}$ maps a surface $S$ of $F_{1}$ into its infinite Nielsen extension and it is the identity on $F_{0}$. To prove the theorem it is necessary to show that

$$
f_{\infty}\left(R^{k} \times(0, \infty)^{n}\right)=R^{k} \times[0]^{n}
$$

i.e. that every punctured surface of $F_{0}$ is the infinite Nielsen extension of some surface with $n$ boundary curves of positive lengths.

It will now be shown that the function $f_{\infty}$ is also continuous on $F_{1}$. It is sufficient to show that given $\varepsilon>0$ and $\delta>0$, then for every simple closed geodesic of length $x, x \leq \delta$, there exists an $N$ such that for all $n \geq N,\left|x_{n}-x_{\infty}\right|<\varepsilon$. Only geodesics not homotopic to boundary curves need to be considered. Suppose that $\varepsilon$ and $\delta$ are given. Pick $N$ such that $\left(1-c_{N}\right) \delta<\varepsilon$ where $c_{N}$ is the constant of inequality II. Then, for all $n \geq N$ and $x<\delta$,

$$
\left|x_{n}-x_{\infty}\right|<\left(1-c_{N}\right) \delta<\varepsilon
$$

To show that $f_{\infty}$ is continuous everywhere, it is only necessary to show that if $S_{m} \rightarrow S_{0}$ where $\left\{S_{m}\right\} \subset F_{1}$ and $S_{0} \in F_{0}$ then $f_{\infty}\left(S_{m}\right) \rightarrow f_{\infty}\left(S_{0}\right)$. This is sufficient because $f_{\infty}$ is continuous on $F_{1}$ and is the identity on $F_{0}$. Since $f_{\infty}\left(S_{0}\right)=S_{0}$, it must be shown that $f_{\infty}\left(S_{m}\right) \rightarrow S_{0}$. It is sufficient to show that, for $m$ large, for every simple closed geodesic of $S_{m}$ of length $x_{m}, x_{m}$ is close to $\left(x_{m}\right)_{\infty}$. But since $S_{m} \rightarrow S_{0}$ and $S_{0} \in F_{0}$, the lengths of the boundary curves of $S_{m}$ must be very small for large $m$. Thus, by inequality II, $x_{m}$ is close to $\left(x_{m}\right)_{\infty}$. Therefore, $f_{\infty}$ is continuous on all of $F$ and the function $f$ satisfies the hypothesis of the lemma. But then $f\left(R^{k} \times(0, \infty)^{n}\right)$ is surjective onto $R^{k} \times[0]^{n}$ and the theorem is proved.

REMARK. After modifying the lemma slightly it can be shown that given a surface $S$ of type $(g ; n, 0)$ there exists an $l$ such that there is a surface of genus $g$ with $n$ holes, each of length $l$, whose infinite Nielsen extension is $S$.

ACKNOWLEDGMENT. I would like to thank Frederick P. Gardiner for suggesting the use of Theorems A and B and for his assistance in formulation of the lemma. I would also like to thank Jacob Sturm for his helpful advice. 


\section{REFERENCES}

1. L. Bers, Nielsen extensions of Riemann surfaces, Ann. Acad. Sci. Fenn. Ser. A I Math. 2 (1976), 17-22.

2. L. Bers and F. Gardiner, A metric for Fricke space which is invariant under the modular group, Adv. in Math. (to appear).

3. N. Halpern, Some remarks on Nielsen extensions of Riemann surfaces, Michigan Math. J. 30 (1983), 65-68.

4. _ On the infinite Nielsen extension of a torus, Complex Variables: Theory and Application 4 (1984), 111-117.

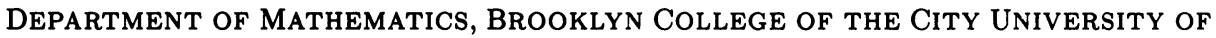
NEW YORK, BROOKLYN, NEW YORK 11210 